ISSN 0103-5150

Fisioter. Mov., Curitiba, v. 26, n. 2, p. 395-402, abr./jun. 2013

Licenciado sob uma Licença Creative Commons

\title{
Análise histomorfométrica do músculo sóleo de ratos submetidos ao modelo animal de parkinsonismo induzido pelo MPTP
}

\author{
Histomorphometrical analysis of rats' soleus muscle submitted \\ to animal model of MPTP-induced parkinsonism
}

\section{Ana Paula Cunha Loureiro ${ }^{[a]}$, Cecília Beatriz Helm Niederheitmann ${ }^{[b]}$, Anna Raquel Silveira Gomes ${ }^{[c]}$, Claudia da Cunha Loureiro ${ }^{[\mathrm{d}]}$, Talita Gianello Gnoato Zotz ${ }^{[\mathrm{e}]}$}

[a] Fisioterapeuta, doutoranda em Ciências da Saúde pela Pontifícia Universidade Católica do Paraná (PUCPR), docente do curso de Fisioterapia da Pontifícia Universidade Católica do Paraná (PUCPR), Curitiba, PR - Brasil, e-mail: anna.loureiro@gmail.com

[b] Mestre em Morfologia pela Universidade Federal do Paraná (UFPR), docente do Setor de Ciências Biológicas da Universidade Federal do Paraná (UFPR), Curitiba, PR - Brasil, e-mail: cecinieder@ufpr.br

[c] Doutora em Ciências Fisiológicas pela Universidade Federal de São Carlos (UFSCar), docente do curso de Fisioterapia da Universidade Federal do Paraná (UFPR) - Setor Litoral e do Programa de Mestrado/Doutorado em Educação Física da Universidade Federal do Paraná (UFPR), Matinhos/Curitiba, PR - Brasil, e-mail: anna.gomes@gmail.com

[d] Bióloga pela Pontifícia Universidade Católica do Paraná (PUCPR), Citotécnica no Laboratório Municipal de Curitiba, Curitiba, PR - Brasil, e-mail: cllaudiacunha@gmail.com

[e] Fisioterapeuta, mestre em Tecnologia em Saúde pela Pontifícia Universidade Católica do Paraná (PUCPR), doutoranda em Educação Física na Universidade Federal do Paraná (UFPR), Curitiba, PR - Brasil, e-mail: talita.gnoato@gmail.com

\section{Resumo}

Introdução: Modelos experimentais da Doença de Parkinson (DP) que reproduzem a desnervação dopaminérgica têm sido desenvolvidos para estudar a patofisiologia desta doença e analisar a eficácia de novas terapêuticas. Dentre os sinais cardinais da DP temos a rigidez muscular, estudos sugerem que mudanças intrínsecas nas propriedades mecânicas do músculo podem ser responsáveis pelo aumento dessa alteração tônica. Objetivo: Analisar a morfologia geral e a histomorfometria do músculo sóleo de ratos Wistar induzidos ao Parkinsonismo por 1-metil-4-fenil-1,2,3,6-tetrahidropiridina (MPTP). Materiais e métodos: Utilizaram-se 24 ratos Wistar machos, com idade de 13 semanas e peso de $279 \pm 13$ g, divididos em quatro grupos: 1 - controle-controle $(n=6)$ : sham tratados com benserazida + salina; 2 - controle-L-DOPA $(n=6)$ : sham tratados com benserazida + L-DOPA; 3 - MPTP- controle $(n=6)$ : lesão na substância negra (SNc) por MPTP tratados com 
benserazida + salina; 4 - MPTP-L-DOPA ( $\mathrm{n}=$ 6): lesão na SNc por MPTP tratados com benserazida + L-DOPA. Esses animais foram submetidos a eutanásia 35 dias após os procedimentos experimentais. Foram analisados: peso corporal, peso muscular, morfologia geral do músculo com microscopia de luz e mensuração da área de secção transversa das fibras musculares. Realizaram-se comparações com o teste $t$ pareado entre o peso corporal inicial e final. A ANOVA post-hoc Tukey foi usada para comparações entre os grupos, sendo considerado significativo $\mathrm{p} \leq 0,05$. Resultados: Não foram encontradas diferenças estatisticamente significativas nas variáveis analisadas. Conclusão: Os dados analisados não excluem a possibilidade de alterações ocorrerem no interior dessas células, nos tipos de fibras musculares ou em longo prazo.

Palavras-chave: Rigidez muscular. Músculo esquelético. Doença de Parkinson.

1-Metil-4-Fenil-1,2,3,6-Tetra-Hidropiridina.

\section{Abstract}

Introduction: Animal models for Parkinson's disease (PD) which mimetize dopaminergic degeneration of nervous cells have been developed to study the pathology of this disease and to analyze the efficiency of new therapies. One of the cardinal signs of PD have muscle stiffness, studies suggest that intrinsic changes in mechanical properties of muscle may be responsible for the increase of this amendment tonic. Objective: To analyze the morphology and histomorphometry of soleus muscle of rats PD-induced by 1-methyl-4-phenyl-1,2,3,6-tetrahydropiridine (MPTP). Materials and methods: We used 24 male Wistar rats, with the age of 13 weeks and $279 \pm 13 \mathrm{~g}$ weight, divided into four groups: 1- control-control ( $n=6)$ : sham treated with benserazidasalina; 2 - control-L-DOPA $(n=6)$ : sham treated with benserazida + L-DOPA; $3-M P T P$-control $(n=6)$ : injury in substantia nigra $(S N c)$ by MPTP treated with benserazidasalina; $4-$ MPTP-L-DOPA ( $n=6)$ : CNS injury by MPTP treated with benserazida $+L$-DOPA. These animals were euthanized 35 days after the experimental procedures. It was analyzed: body weight, muscle weight, General muscle morphology with light microscopy and measurement of the cross-sectional area of muscle fibers. Comparisons between initial and final body weight were developed with the paired T-test. The Tukey post-hoc ANOVA was used for comparisons between groups, being considered significant $p \leq 0.05$. Results: No statistically significant differences were found in the analyzed variables. Conclusion: The data analyzed do not exclude the possibility of occurrence of changes in the interior of these cells, in the types of muscle fibers or long term.

Keywords: Muscle rigidity. Skeletal muscle. Parkinson Disease. 1-Methyl-phenyl-1,2,3,6-tetrahydropyridine.

\section{Introdução}

A Doença de Parkinson (DP), também conhecida como paralisia agitante ou síndrome parkinsoniana idiopática, é uma doença crônica e progressiva $(1,2)$. Juntamente com a Doença de Alzheimer é uma das mais comuns desordens neurodegenerativas relacionadas à idade em humanos e constitui-se em um dos distúrbios do movimento mais encontrados na população idosa, entre 50 e 70 anos de idade. Os distúrbios do movimento resultantes caracterizam-se por tremores, rigidez, bradicinesia e instabilidade postural $(2,3)$.

No início da evolução da doença, a morte de alguns neurônios não causa diferença significativa na transmissão do sinal. Como a doença é evolutiva, a progressão da morte neuronal causa diminuição importante na concentração de dopamina (DA) junto aos receptores estriatais. Como resposta, os neurônios estriatais sintetizam e expõem mais receptores na membrana plasmática, aumentando a eficiência da sinapse e mascarando os efeitos da depleção. $\mathrm{Na}$ fase mais avançada da doença, essa regulação positiva de receptores não é o suficiente para manter a conexão em níveis normais, causando deficit motor (4). Quando o paciente parkinsoniano começa a apresentar os sinais motores da doença, já perdeu mais de 70\% dos neurônios nigroestriatais. Com isso, fica muito difícil reverter o quadro, o que faz com que a maioria dos tratamentos, tanto cirúrgicos como farmacológicos, reduzam a sintomatologia, não permitindo um prognóstico de cura (2). A progressão dos sintomas ocorre lentamente, porém a velocidade com que a progressão se desenvolve varia de indivíduo para indivíduo. As principais manifestações são as motoras, porém evidências clínicas e experimentais 
indicam que essa desordem neurodegenerativa também causa deficit cognitivos $(5,6)$.

A rigidez muscular é um dos primeiros sinais importantes do parkinsonismo. É uma resistência aumentada no movimento passivo, afetando toda a musculatura estriada e ocorre em toda amplitude de movimento. A rigidez pode ser distribuída desigualmente, iniciando frequentemente em um membro, ou um dos lados, podendo se disseminar em todo o corpo com a evolução da doença. 0 movimento de balanço normal dos braços está ausente durante a marcha devido à rigidez do tronco. 0 movimento ativo, concentração mental ou tensão emocional podem aumentar a intensidade da rigidez. Pode ser do tipo roda dentada, quando no movimento passivo a resistência é intermitente; e a rigidez do tipo cano de chumbo, quando a resistência é uniforme e regular, sem flutuações para o movimento passivo (7).

0 dano neurológico resultará em impedimentos do sistema musculoesquelético decorrentes da rigidez, desequilíbrio muscular, acinesia e bradicinesia. Entretanto, considerando que o impedimento primário das lesões ocorridas nos núcleos da base é o deficit de planejamento e de programação de movimento, a rigidez é tratada como compensação (8).

Modelos experimentais da DP que reproduzem a desnervação dopaminérgica têm sido desenvolvidos para estudar a patofisiologia da doença e para analisar a eficácia de novas terapêuticas. A DP é uma doença humana e não se manifesta espontaneamente em animais, é somente observada através da administração de agentes neurotóxicos que interrompam ou destruam seletivamente o sistema catecolaminérgico, tais como a 6-hidroxidopamina (6-OHDA), a metanfetamina, e a 1-metil-4-fenil-1, 2, 3,6-tetrahidropiridina (MPTP) $(9,10)$. Ao comparar os agentes neurotóxicos que induzem a DP em animais, verificou-se que a administração de MPTP em macacos é o melhor modelo de discinesias o que contribui para o avanço de novas técnicas de tratamento (11). Contudo, a utilização desse modelo animal demanda altos custos e não são todos os centros de pesquisas que trabalham com esse modelo; dessa forma, a utilização de ratos como modelo animal para DP tem se mostrado eficaz na reprodução das discinesias características da doença (11). Além disso, foi demonstrado que a administração de MPTP em ratos mimetiza a fase inicial da DP (12).

Dessa forma, o objetivo desse estudo foi verificar se a lesão seletiva e parcial da substância negra (SNc) de ratos Wistar por MPTP causa alteração na morfologia do músculo sóleo ainda na fase inicial da DP.

\section{Materiais e métodos}

Foi realizado estudo experimental, com ratos Wistar Albino induzidos ao modelo de Parkinsonismo por 1-methyl-4-phenyl-1,2,3,6-tetrahydropyridine (MPTP), o qual foi desenvolvido no Centro de Ciências Biológicas da Universidade Federal do Paraná.

Foram utilizados 24 ratos da linhagem Wistar com idade de 13 semanas e peso de $279 \pm 13$ g obtidos de um experimento científico do laboratório do Departamento de Farmacologia da UFPR, aprovado pelo Comitê de Ética em Pesquisa da UFPR de número 218, processo 23075.016767/2007-67 e RO $04 / 2007$ - 08/05/2007. Esses animais foram divididos em quatro grupos (Tabela 1).

\section{Procedimento cirúrgico experimental}

Na cirurgia estereotáxica, os animais lesados na SNc receberam infusão bilateral de $1 \mu \mathrm{l}$ da toxina 1-metil-4-fenil-1,2,3,6-tetrahidropiridina (MPTP) $(100 \mu \mathrm{g})$. Os animais do grupo controle foram submetidos ao mesmo procedimento, porém receberam infusão de salina em vez de neurotoxina. Após o procedimento, os animais foram suturados e receberam $1 \mathrm{ml}$ de bicarbonato se sódio saturado, para acelerar a eliminação do anestésico, e foram mantidos na sala com temperatura controlada para recuperação pós-anestésica; logo em seguida, retornaram para suas gaiolas onde foram mantidos durante todo o protocolo experimental. Os animais foram agrupados em controle (sham operados) e lesados na SNc bilateralmente, para os quais a toxina escolhida foi o 1-metil-4-fenil-1,2,3,6-tetra-hidropiridina (MPTP) $(11,12)$.

Após 35 dias de lesão, os animais foram submetidos a eutanásia seguida do processo para a dissecação do músculo sóleo da pata esquerda. Os músculos foram periodicamente gotejados com solução salina $(\mathrm{NaCl} 0,9 \%)$ para evitar ressecamento tecidual. Em seguida cada músculo foi pesado isoladamente com uso de uma balança analítica de precisão (Mettler/Toledo com capacidade mínima $10 \mathrm{mg}$ e máxima $210 \mathrm{~g}$ ). Os músculos foram estendidos em uma superfície plana (cortiça) e fixados pelas extremidades com agulhas de acupuntura facial para 
evitar retração do material e colocado em solução de formalina tamponada.

0 fragmento retirado da metade medial do ventre de cada músculo sóleo foi corado com Hematoxilina de Harris e Eosina (HE). De todos os músculos foram obtidos cortes histológicos $(4 \mu \mathrm{m})$ transversais e seriados, em micrótomo (Leica) para avaliação da morfologia geral.

Para análise histomorfométrica da área de secção transversa das fibras musculares foram realizadas fotomicrografias dos cortes histológicos em microscópio de luz (Axyophot, Carl Zeiss, Oberkochen, Germany) e capturadas em sistema de videoimagem (Applied Spectral Imaging, Migdal Ha'emek, Israel) através do Programa Case Data Manager Expo (Applied Spectral Imaging, Migdal Ha'emek, Israel, versão 4.0). Em cada músculo, foi medida a área de secção transversa das fibras musculares, com o auxílio do software UTHSCSA Image Tool 3.0 (desenvolvido na University of Texas Health Science Center at San Antonio, Texas; e disponível no endereço http://ddsdx.uthscsa.edu/dig/itdesc.html) (13).

Foi mensurada a área de secção transversa de 100 fibras musculares de cada músculo, escolhida de modo aleatório da região do ventre muscular da secção histológica, como descrito por Coutinho (14).

\section{Análise estatística}

As variáveis analisadas: peso corporal ( $t$-Student), peso muscular e área de secção transversa das fibras musculares foram avaliadas pela comparação entre os resultados obtidos nos músculos das patas de cada animal em cada grupo experimental. Os resultados foram também comparados entre os grupos experimentais por meio da ANOVA post-hoc Tukey. Os valores foram considerados significativos quando $\mathrm{p} \leq 0,05$.

Tabela 1 - Divisão dos grupos experimentais

\begin{tabular}{|c|c|c|}
\hline Grupo & Designação (abreviação) & Ratos (n) \\
\hline Controle (ratos sham tratados com benserazida + salina) & $S H A M+S A L$ & 6 \\
\hline $\begin{array}{l}\text { Controle - L-DOPA (ratos sham tratados com benserazida + } \\
\text { L-DOPA) }\end{array}$ & SHAM + L-DOPA & 6 \\
\hline $\begin{array}{l}\text { MPTP (ratos com lesão na SNc por MPTP tratados com } \\
\text { benserazida + salina) }\end{array}$ & MPTP + SAL & 6 \\
\hline $\begin{array}{l}\text { MPTP - L-DOPA (ratos com lesão na SNc por MPTP tratados } \\
\text { com benserazida + L-DOPA) }\end{array}$ & MPTP + L-DOPA & 6 \\
\hline
\end{tabular}

Fonte: Dados da pesquisa.

\section{Resultados}

Em relação ao peso corporal nenhum dos quatro grupos de animais mostrou alteração significativa dessa variável, quando comparado com o peso inicial. Os animais do grupo sham + salina $(298 \pm 12 \mathrm{~g} v$. $294 \pm 26 \mathrm{~g}, \mathrm{p}=0,090$, teste $t$ pareado); MPTP + sal $(268 \pm 15 \mathrm{~g} v s .264 \pm 38 \mathrm{~g}$, $\mathrm{p}=0,150$, teste $t$ pareado); sham + L-DOPA $(293 \pm 18$ g vs. $287 \pm 34$ g, $p=0,211$, teste $t$ pareado) e MPTP + L-DOPA $(276 \pm 11 \mathrm{~g} v s .271 \pm$ $48 \mathrm{~g}, \mathrm{p}=0,477$, teste $t$ pareado). Na comparação entre os grupos também não se observou diferença significativa (Figura $1 \mathrm{~A}$ ).

0 peso muscular dos quatro grupos de animais não mostrou modificação significativa na comparação entre os grupos (ANOVA). A média de peso dos músculos sóleos de cada grupo foi: sham + salina $(0,126 \pm$ $0,018 \mathrm{~g}) ; \mathrm{MPTP}+$ sal $(0,121 \pm 0,008 \mathrm{~g}) ;$ sham + L-DOPA $(0,129 \pm 0,013 \mathrm{~g})$ e MPTP + L-DOPA $(0,125 \pm$ $0,011 \mathrm{~g}$ ) (Figura 1B).

Nenhum dos quatro grupos de animais mostrou alteração na área de secção transversa das fibras musculares do músculo sóleo quando comparada entre os grupos (ANOVA). Os resultados encontrados em cada grupo foram: grupo sham + sal (2245 \pm $\left.815 \mu \mathrm{m}^{2}\right) ;$ MPTP + sal $\left(3145 \pm 1271 \mu \mathrm{m}^{2}\right) ;$ sham + L-DOPA (2904 $\left.\pm 653 \mu \mathrm{m}^{2}\right)$ e MPTP + L-DOPA (2451 \pm $623 \mu^{2}$ ) (Figura 1C).

$\mathrm{Na}$ análise morfológica não foram encontrados sinais histopatológicos. 

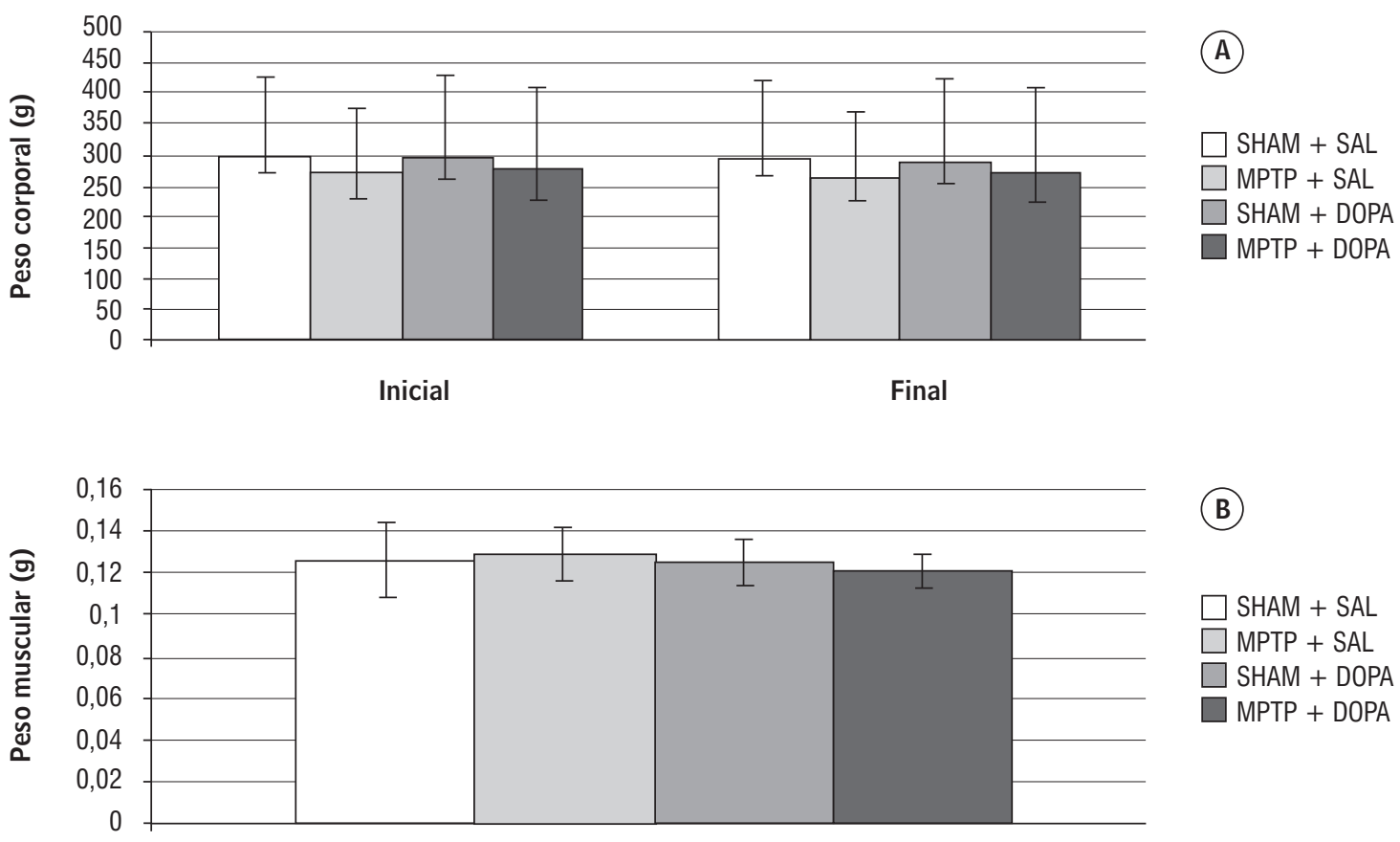

(B)
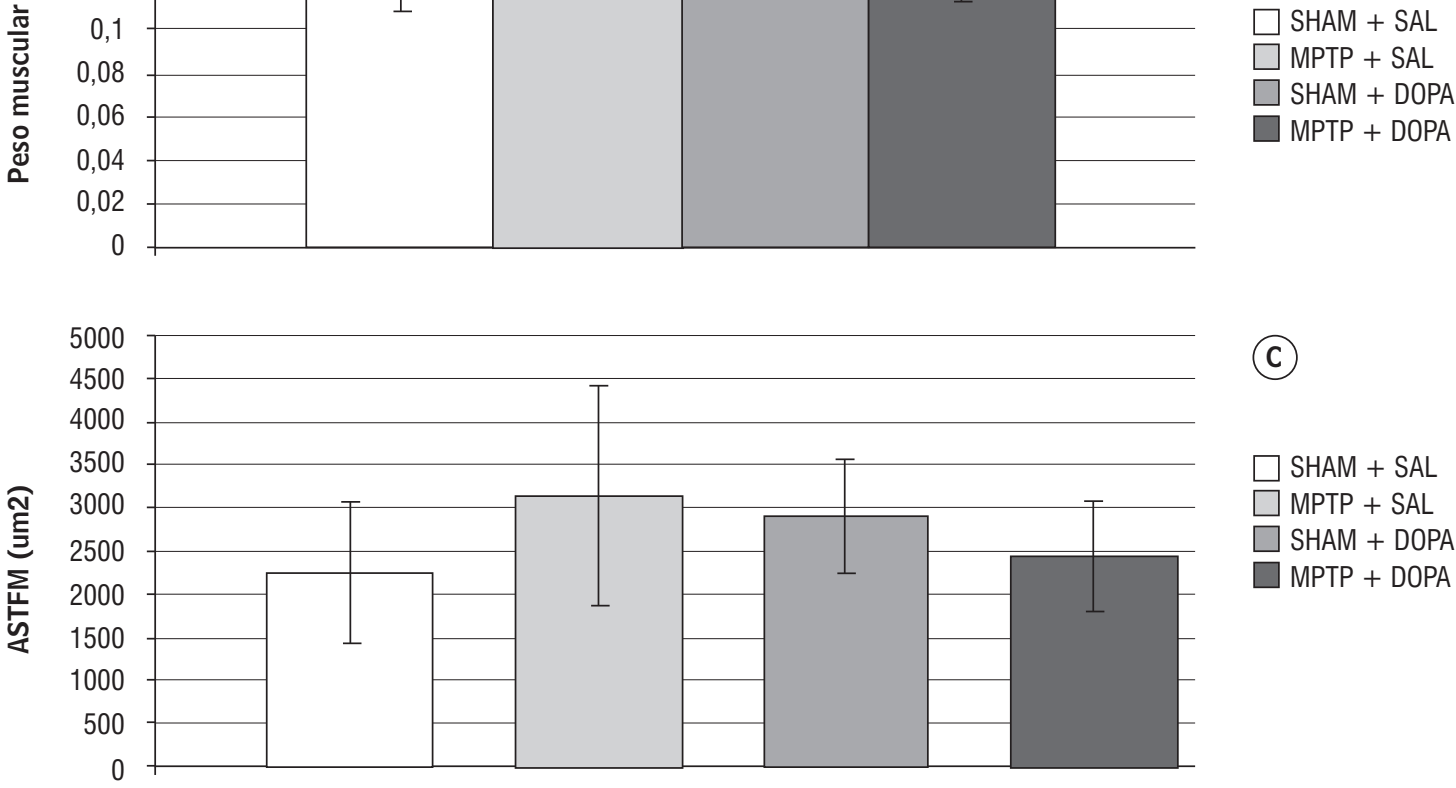

(C)

$S H A M+S A L$

$\square$ MPTP + SAL

$\square$ SHAM + DOPA

MPTP + DOPA

Gráfico 1- Peso corporal (A), peso muscular (B), área de secção transversa das fibras (C)

Legenda: SHAM + SAL = ratos sham tratados com benserazida e salina; MPTP + SAL = ratos com lesão na SNc por MPTP tratados com benserazida + salina; SHAM + L-DOPA: ratos sham tratados com benserazida e L-DOPA; MPTP + L-DOPA = ratos com lesão na SNc por MPTP tratados com benserazida + L-DOPA.

Fonte: Dados da pesquisa.

Nota: Os resultados estão representados como a média \pm desvio padrão.

\section{Discussão}

A DP é caracterizada principalmente pela degeneração das células da camada ventral da parte compacta da substância negra (SNc) e do locus ceruleus. A patologia inclui a identificação dos corpos de Lewy, que são inclusões eosinófilcas intracitoplasmáticas constituídas por várias estruturas proteicas, em neurônios remanescentes na SNc (5).

Os neurônios dopaminérgicos da SNc são particularmente vulneráveis ao processo neurodegenerativo que ocorre na DP. A degeneração dessas células resultará na diminuição da produção de DA com consequente disfunção da via nigroestriatal (2). 
Podem ocorrer alterações significativas nos receptores de DA no corpo estriado, resultando numa queda nas ligações de DA nos gânglios de base. A quebra nas ligações dopaminérgicas resulta num desequilíbrio nos sistemas mutuamente antagonistas dos gânglios de base. 0 sistema colinérgico, atuando por meio do seu neurotransmissor acetilcolina, teoricamente permite a atividade dos interneurônios de axônios curtos do corpo estriado, e acredita-se que o sistema dopaminérgico propicie a inibição tônica desses interneurônios colinérgicos. Quando há carência de dopamina, a excessiva resposta excitatória resulta numa ativação generalizada dos sistemas motores esquelético e intrafusal por meio dos tratos corticoespinhal, reticuloespinhal e rubroespinhal (15).

Para obter um modelo animal da DP, a partir do uso do MPTP, devem ser considerados o método de administração e a espécie animal a ser utilizada. Os animais mais usados para estudos do MPTP são camundongos e macacos. Por ser um composto altamente lipofílico, o MPTP, após sua administração sistêmica, atravessa facilmente a barreira hematoencefálica, atingindo rapidamente o sistema nervoso central $(2,16)$.

Nesse estudo foram investigados grupos de ratos induzidos ao modelo de parkinsonismo pelo MPTP com e sem tratamento medicamentoso, comparados a grupos de ratos placebo também com e sem tratamento medicamentoso.

Não foi identificada diferença no peso muscular, na área de secção transversa, tampouco na morfologia geral das fibras. Assim, os resultados encontrados nesse modelo animal de Parkinson induzido expressaram a fase inicial da doença, mostrando padrões de normalidade histomorfométrica muscular esquelética. Talvez, tais achados justifiquem-se pelo fato de que os maiores sinais motores da Doença de Parkinson começam a se manifestar somente após perda de mais de $70 \%$ dos neurônios nigroestriatais, caracterizando fase avançada da doença (2).

No entanto, esse estudo justifica-se pela ausência de descrições na literatura sobre a morfologia do músculo esquelético antes da instalação do quadro de rigidez em ratos com DP induzida. Além disso, não foi encontrada nenhuma pesquisa que investiga a histologia do músculo esquelético com rigidez muscular em modelo animal para fins comparativos.

Rossi et al. (17), em um estudo com humanos em que se compararam as mudanças na velocidade de condução da fibra muscular, a frequência média e a biopsia muscular entre indivíduos com DP e hígidos na mesma faixa etária, apontaram uma tendência à hipertrofia das fibras do tipo I e, em alguns casos, atrofia de fibras do tipo II nos indivíduos com DP. Esse estudo sugere que alterações nas propriedades mecânicas intrínsecas do músculo seriam responsáveis pelo aumento da rigidez muscular (18).

Encontramos maior riqueza de estudos apontando alterações estruturais e mecânicas em músculos esqueléticos secundariamente à espasticidade como: o aumento variável do tamanho da fibra, aumento do número de fibras "arredondadas", fibras musculares com alterações do tipo "ruídos de traça" e, em alguns casos, aumento do espaço extracelular $(19,20)$.

Lorene-Koci et al. (21) demonstraram em seus estudos a presença de rigidez muscular em ratos induzida por reserpina ou haloperidol. A rigidez aparece em resposta à movimentação passiva e essa resistência muscular é caracterizada pelo aumento da atividade reflexa registrada pela eletromiografia. Injeções bilaterais de N-ethoxycarbonyl-2-ethoxy1,2-dihydroquiline (EEDQ) também causaram rigidez muscular evidenciada pela eletromiografia (22).

Modelo de rigidez semelhante à DP também foi encontrada em ratos quatro semanas após o uso da neurotoxina 6-OHDA induzindo lesão da substância negra, constatada pela mecanografia e eletromiografia. Entretanto, ratos jovens, apesar da alta percentagem da lesão, apresentaram mecanismos compensatórios que parecem ser altamente eficazes para compensar os efeitos degenerativos; por exemplo, aumento da síntese de dopanima nos neurônios sobreviventes, proliferação de neurônios dopaminérgicos e brotamentos de axônios colaterais (23).

Lindner et al. $(24,25)$ procuraram em seu estudo determinar se sinais clínicos parkinsonianos poderiam ser detectados em ratos com perda de células nigroestriatais e depleção dos níveis de dopamina, e se ratos de meia-idade (12 meses) teriam mais deficit que ratos jovens ( 2 meses). Os animais receberam infusão estriatal bilateral de 6-OHDA e foram avaliados após três meses de lesão. Encontrou-se, mesmo com essa lesão incompleta das células da via nigroestriatal e redução dos níveis de dopamina estriatal, uma clínica plena: acinesia, rigidez muscular, tremor, deficit visuoespacial e cognitivo. Os resultados também mostraram que os ratos mais velhos apresentaram condições clínicas mais semelhantes aos sintomas parkinsonianos. 
A cirurgia estereotáxica permitiu a demonstração de que a infusão de MPTP diretamente na SNc de ratos produz lesão significativa de neurônios, porém, de forma mais moderada do que a 6-OHDA (25). Os poucos estudos utilizando o MPTP geram dúvidas se esse modelo é válido para o estudo de alterações motoras associadas à DP; por outro lado, ele tem sido amplamente utilizado para estudos dos deficit cognitivos associados a essa doença $(12,13,25)$. Sendo assim, o modelo de lesão da via nigroestriatal com 6-OHDA é utilizado para o estudo das alterações motoras da DP e ação de drogas antiparkinsonianas, já o modelo de lesão da via nigroestriatal com MPTP tem sido utilizado mais para o estudo dos deficit cognitivos (10).

\section{Conclusão}

Não se observaram alterações significativas no peso corporal e muscular, área de secção transversa das fibras musculares ou achados histopatológicos do músculo sóleo de ratos com lesão bilateral induzida pelo modelo com $1 \mu \mathrm{l}$ da toxina MPTP na SNc, reproduzindo a fase inicial da doença de Parkinson, em ratos jovens, por um período de lesão de 35 dias, tratados ou não com o medicamento L-DOPA. No entanto, esses dados não excluem a possibilidade de alterações ocorrerem no interior das células, uma vez que não foram avaliados os tipos de fibras musculares e a ultraestrutura celular, nem foram realizadas em longo prazo investigações musculares em ratos com lesões induzidas.

\section{Referências}

1. Kaufmann TL. Manual de reabilitação geriátrica. Rio de Janeiro: Guanabara Koogan; 2001.

2. Menezes MS, Teive HAG. Doença de Parkinson. 2. ed. Rio de Janeiro: Guanabara Koogan; 2003.

3. Guccione AA. Fisioterapia geriátrica. Rio de Janeiro: Guanabara Koogan; 2002.

4. Obeso JÁ, Rodriguez-Oroz MC, Rodríguez M, Lanciego JL, Artieda J, Gonzalo N, et al. Pathophysiology of the basal ganglia in Parkinson's disease. Trends Neurosci. 2000;23(10Suppl):S8-19. doi:10.1016/ S1471-1931(00)00028-8.
5. Freitas EV, Xavier FA. Tratado de geriatria e gerontologia. Rio de Janeiro: Guanabara Koogan; 2002.

6. da Cunha C, Silva MHC, Wietzikoski S, Wietzikoski EC, Ferro MM, Kouzmine I, et al. Place learning strategy of substantia nigra pars compacta-lesioned rats. Behav Neurosci. 2006;120(6):1279-84. doi:10.1037/0735-7044.120.6.1279.

7. Stokes M. Neurologia para fisioterapeutas. São Paulo: Premier; 2000.

8. Rebelatto JR, Morelli JGS. Fisioterapia geriátrica: a prática da assistência ao idoso. São Paulo: Manole; 2004.

9. Gerlach M, Riederer P. Animal models of Parkinson's disease: an empirical comparison with the phenomenology of the disease in man. J. Neural Transm. 1996;103:987-1041. doi:10.1007/BF01291788.

10. Wietzikoski EC. Estudo comparativo do comportamento ipsiversivo e contraversivo nos modelos da 6-OHDA e MPTP da doença de Parkinson [dissertação]. Curitiba: Mestrado em Farmacologia, Universidade Federal do Paraná; 2006.

11. Morin N, Jourdain VA, Di Paolo T. Modeling dyskinesia in animal models of Parkinson disease. Exp Neurol. 2013. doi:10.1016/j.expneurol.2013.01.024.

12. Ferro MM, Bellissimo MI, Anselmo-Franci JA, AngelluccI ME, Canteras NS, da Cunha C. Comparison of bilaterally 6-OHDA- and MPTP-lesioned rats as models of the early phase of Parkinson's disease: histological, neurochemical, motor and memory alterations. J. of Neuros. Meth. 2005;148(1):78-87. doi:10.1016/j. jneumeth.2005.04.005.

13. Secchi KV, Morais CP, Cimatti PF, Tokars E, Gomes ARS. Efeito do alongamento e do exercício contra-resistido no músculo esquelético de rato. Rev Bras Fisioter. 2008;12(3):228-34.

14. Coutinho EL, Gomes ARS, França CN, Oishi J, Salvini TF. Effect of passive stretching on the immobilized soleus muscle fiber morphology. Braz. J. of Med. and Biol Res. 2004;37:1853-61. doi:10.1590/ S0100-879X2004001200011.

15. O'Sullivan SB, Schmitz TJ.Fisioterapia: avaliação e tratamento.2. ed. Rio de Janeiro: Manole; 2004. 
16. Lorenc-Koci E, Smialowska M, Antkiewicz-Michaluk L, Golembiowska K, Bajkowska M, Wolfarth S. Effect of acute and chronic administration of 1,2,3,4-tetrahydroisoquinoline on muscle tone, metabolism of dopamine in the striatum and tyrosine hydroxylase immunocytochemistry in the substantia nigra, in rats. Neuros. 2000;95:1049-59. doi:10.1016/ S0306-4522(99)00511-4.

17. Rossi B, Siciliano G, Carboncini MC, Manca ML, Massetani R, Viacava P, et al. Muscle modifications in Parkinson's disease: myoelectric manifestations. Neurosci Lett. 2002;317(3): 23-6.

18. Dietz V, Quitern J, Berger W. Electrophysiological studies of gait in spasticity and rigidity: evidence that altered mechanical properties of muscle contribute to hypertonia. Brain. 1981;104:431-49. doi:10.1093/ brain/104.3.431.

19. Foran JRH, Steinman S, Chambers HG, Lieber RL. Structural and mechanical alterations in spastic skeletal muscle. Develop. Medic. \& Child Neurol. 2005;47:713-17. doi:10.1017/S0012162205001465.

20. Lieber RL, Steinman S, Barash IA, Chambers H. Structural and functional changes in spastic skeletal muscle. Musc. \& Nerv. 2004;29:615-27. doi:10.1002/ mus.20059.

21. Lorenc-Koci E, Wolfarth S, Ossowska K. Haloperidolincreased muscle tone in rats as a model of parkinsonian rigidity. Exp. Brain Res. 1996;109:268-76. doi:10.1007/BF00231786.
22. Hemsley KM, Farral EJ, Crocker AD. Dopamine receptors in the subthalamic nucleus are involved in the regulation of muscle tone in the rat. Neurosc. Lett. 2002;317:123-26. doi:10.1016/ S0304-3940(01)02460-0.

23. Wolfarth S, Konieczny J, Smialowska M, Schulze G, Ossowska K. Influence of 6-hydroxydopamine lesion of the dopaminergic nigrostriatal pathway on the muscle tone and electromyographic activity measured during passive movements. Neurosc. 1996;74:985-96.

24. Lindner MD, Cain CK, Plone MA, Frydel BR, Blaney TJ, Emerich DF, et al. Incomplete nigrostriatal dopaminergic cell loss and partial reductions in striatal dopamine produce akinesia, rigidity, tremor and cognitive deficits in middle-aged rats. Behav Brain Res. 1999;102:116. doi:10.1016/S0166-4328(98)00160-0.

25. da Cunha C, Gevaerd MS, Vital MABF, Miyoshi E, Andreatine R, Silveira R, et al. Memory disruption in rats with nigral lesions induced by MPTP: a model for early Parkinson's disease amnesia. Behav. Brain Res. 2001;124:9-18. doi:10.1016/ S0166-4328(01)00211-X.

Recebido: 16/09/2012 Received: 09/16/2012

Aprovado: $13 / 04 / 2013$ Approved: 04/13/2013 